\title{
Stone crayfish in the Czech Republic: how does its population density depend on basic chemical and physical properties of water?
}

\author{
P. Vlach ${ }^{(1) \star}$, J. Svobodová(2), D. Fischer ${ }^{(3)}$ \\ Received July 20, 2012
}

Revised November 11, 2012

Accepted December 17, 2012

\begin{abstract}
Key-words: the stone crayfish, physical and chemical parameters of water, abundance, the Czech Republic

The stone crayfish (Austropotamobius torrentium Schrank) is one of the two native crayfish species in the Czech Republic. The populations as well as physical and chemical parameters of water $(\mathrm{pH}$, conductivity, dissolved oxygen, undissolved particles, $\mathrm{NH}_{3}, \mathrm{NH}_{4}^{+}, \mathrm{NO}_{2}^{-}, \mathrm{NO}_{3}^{-}$, phosphorus, $\mathrm{Ca}^{2+}$ and $\mathrm{SO}_{4}^{2-}$ ) of 33 streams were examined to find the ecological plasticity of this crayfish and some relations between these parameters and population densities. The mentioned parameters often significantly varied at the sites. Two approaches were applied to find relations between these parameters and observed abundance. At first, the observed streams were compared using RDA (streams $\times$ physical-chemical parameters). No significance was found while testing relationship between the streams grouped along the 1st axis of model and the observed abundances of stone crayfish. However, some correlations between abundance and conductivity, calcium, nitrates and sulphates were found using polynomial regression. These relationships are explicable in terms of mutual correlations, underlying geology and other factors which affect abundances. In conclusion, A. torrentium is able to inhabit waters with a large range of physical and chemical parameters of the water without any fundamental influence on population densities. Water properties play an indisputable role as limiting ecological factors at uncommon concentrations, but population densities are probably influenced much more by the types of habitats, habitat features, predation and other ecological factors.
\end{abstract}

\section{RÉSUMÉ}

L'écrevisse des torrents en République tchèque : comment la densité de sa population dépend des propriétés physico-chimiques de l'eau?

\begin{abstract}
Mots-clés : écrevisse des torrents, physico-chimie de l'eau

L'écrevisse des torrents (Austropotamobius torrentium Schrank) est l'une des deux espèces d'écrevisses indigènes de la République tchèque. Les populations ainsi que les paramètres physiques et chimiques de l'eau $(\mathrm{pH}$, conductivité, oxygène dissous, particules en suspension, $\mathrm{NH}_{3}, \mathrm{NH}_{4}^{+}, \mathrm{NO}_{2}^{-}, \mathrm{NO}_{3}^{-}$, le phosphore, $\left.\mathrm{Ca}^{2+}, \mathrm{SO}_{4}^{2-}\right)$ de 33 cours d'eau ont été examinés pour déterminer la plasticité écologique de cette écrevisse et des relations entre ces paramètres et les densités de population. Les paramètres étudiés varient souvent beaucoup suivant les sites. Deux approches ont été appliquées pour chercher des relations entre ces paramètres et l'abondance observée.
\end{abstract}

(1) Faculty of Education of the University of West Bohemia, Department of Biology, Klatovská 51, 30619 Plzeň, Czech Republic

(2) G. Masaryk Water Research Institute, Podbabská 30, 16062 Prague 6, Czech Republic

(3) Mining Museum Přibram, Nám. Hynka Kličky 293, 26101 Přibram VI, Czech Republic

* Corresponding author: vlach.pavel@mybox.cz 
abondance, République Tchèque
Dans un premier temps, les rivières étudiées ont été comparées par une RDA (ruisseaux $\times$ paramètres physico-chimiques). Aucune signification n'a été trouvée en testant la relation entre les rivières regroupées le long du $1^{\mathrm{er}}$ axe de ce modèle et les abondances observées des écrevisses des torrents. Cependant, certaines corrélations entre l'abondance et la conductivité, le calcium, nitrates et sulfates ont été trouvées en utilisant la régression polynomiale. Ces relations sont explicables en termes de corrélations mutuelles, qui révèlent la géologie et d'autres facteurs qui influent sur l'abondance (par exemple la taille des cours d'eau, la compétition, les empoisonnements et la sécheresse). En conclusion, $A$. torrentium est capable de vivre dans les eaux d'une large gamme de paramètres physiques et chimiques sans aucune influence fondamentale sur les densités de population. Les propriétés de l'eau jouent un rôle indiscutable comme facteurs écologiques limitants en cas de concentrations exceptionnelles, mais les densités de population sont sans doute bien plus influencées par le type d'habitat, les caractéristiques de l'habitat, la prédation et d'autres facteurs écologiques.

\section{INTRODUCTION}

Crayfish play an irreplaceable role in freshwater invertebrate communities (Füreder and Reynolds, 2003; Sint and Füreder, 2004; Souty-Grosset et al., 2006) such as various species of macroinvertebrates. Crayfish can play an important role as bioindicators, providing basic information about water quality (Wright et al., 2000).

There are five crayfish species in the Czech Republic, but only two species - the noble crayfish Astacus astacus (Linnaeus, 1758) and the stone crayfish Austropotamobius torrentium (Schrank, 1803) - are considered as indigenous (Holdich, 2002; Kozák et al., 2002; Machino and Füreder, 2005; Souty-Grosset et al., 2006; Vlach et al., 2009b). Both species are listed in the IUCN Red List as vulnerable. Stone crayfish has an IUCN "data deficient" status and belongs among priority species given by the Habitats Directive (European Communities, 1992). Both of the above-mentioned species are protected by law (114/1992) as critically endangered species in the Czech Republic.

Crayfish are endangered by many factors which lead to decrease in population densities or local extinctions. Water quality is often considered as one of the most important factors affecting the presence of crayfish or negatively influencing their population characteristics (Füreder et al., 2003; Holdich, 2002; Seiler and Turner, 2004). There are many contributions describing the influence of water properties, especially toxicity of hard metals, in laboratory conditions (Alexopoulos et al., 2003; Guner, 2007; Jeberg and Jensen, 1994; Kozák et al., 2005; Ward et al., 2006; Yildiz and Benli, 2004). On the other hand, better further studies evaluating crayfish presence/absence in relation to water quality (Bohl, 1987; Broquet et al., 2002; Foster, 1995; Kozák et al., 2002; Pârvulescu et al., 2011; Svobodová et al., 2012; Trouilhé et al., 2007) have been conducted. Some works were unable to find relations between crayfish presence and water quality. Moreover, other studies have considered the type of substratum the main factor delimiting the distribution of crayfish rather than water quality (Lachat and Laurent, 1987).

This study provides data about basic water characteristics of most of the known localities in the Czech Republic inhabited by the stone crayfish; see the enclosed list of the localities given by Vlach et al. (2009b). The main aim of the present paper is to find some connections between population densities of stone crayfish and water physical-chemical characteristics, and to gain information about the ecological demands of this crayfish species. Such knowledge is one of the most important bases for conservation of the endangered species which are often considered "flagship species" and belong to the cultural and natural heritage of European countries (Demers et al., 2006; Füreder et al., 2003; Trouilhé et al., 2007). 


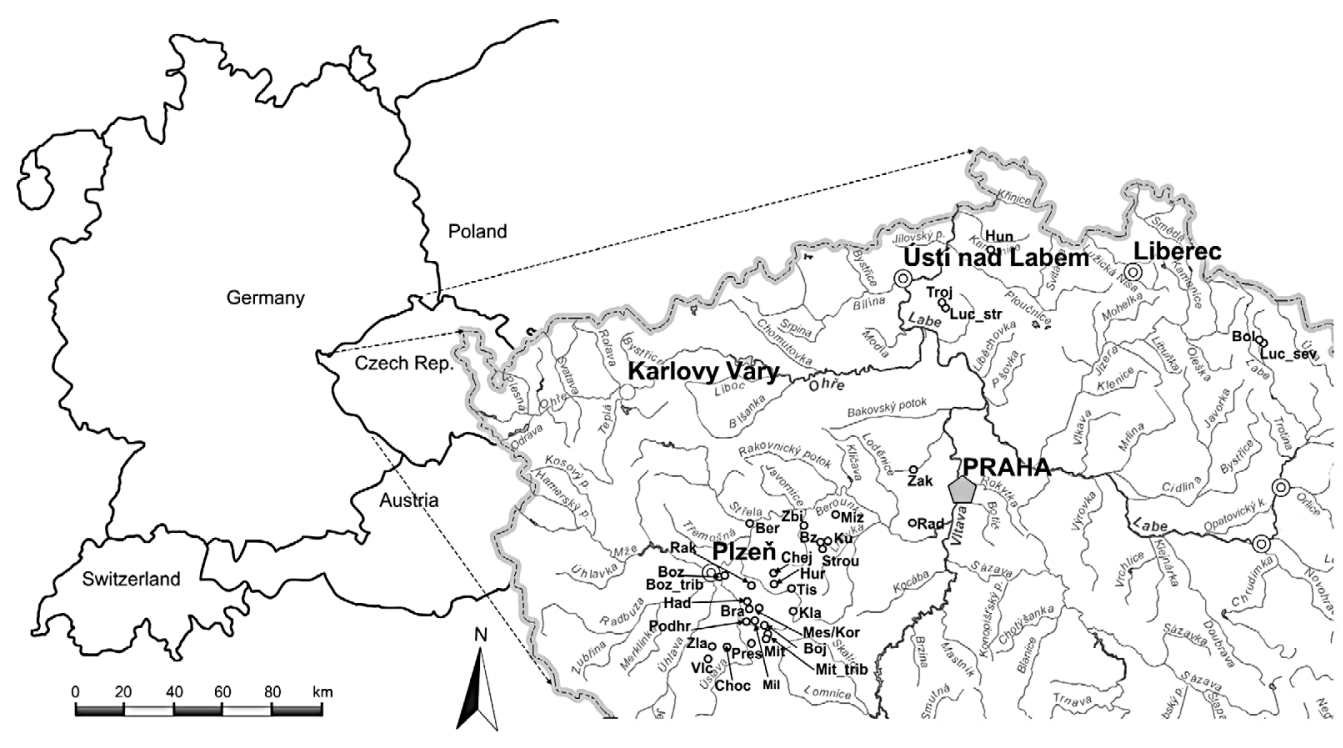

\section{Figure 1}

Map of examined localities of the stone crayfish Austropotamobius torrentium in the Czech Republic. Ber - Bertínský brook, Boj - Bojovka, Bol - Bolkovský brook, Boz - Božkovský brook, Boz_trib - right tributary of the Božkovský brook, Bra - Bradava, Bz - Bzovský brook, Had - Hrádecký brook, Hun Huníkovský brook, Hur - Håurecký brook, Chej - Chejlava (Úzký brook), Choc - Chocenický brook, Kla - Klabava, Kor/Mes - Kornatický/Mešenský brook, Ku - Kublovský brook, Luc_sev - Luční brook (Giant Mts.), Luc_stre - Luční brook (Central Bohemia Highlands), Mil - Milínovský brook, Mit - Mítovský brook, Mit_trib - left tributary of Mítovský brook, Miz - Míza, Podhr - Podhrázský brook, Pres - Přešínský brook, Rad - Radotínský brook, Rak - Rakovský brook, Strou - Stroupínský brook, Tis - Tisý brook, Troj Trojhorský brook and its tributary, VIc - Vlčí (Kbelský) brook, Zak - Zákolanský brook, Zbi - Zbirožský brook, Zla - Zlatý (Př́chovický) brook.

\section{MATERIALS AND METHODS}

\section{> DESCRIPTION OF SAMPLING SITES}

The study was carried out on 33 streams (40 sample sites) out of the 41 streams in the Czech Republic inhabited by stone crayfish. These localities were described in detail by Vlach et al. (2009b). The list of the observed streams and their location in the country are depicted in Figure 1.

\section{> DATA COLLECTION}

Data about abundance of the stone crayfish were obtained from continuous monitoring of the stone crayfish populations from 2007-2010. At each stream, monitoring took place on patches of $30 \mathrm{~m}^{2}$ randomly chosen at each visit. All potential shelters on these patches were examined by hand. Data concerning population density was collected in two periods: MayJune and September-October. No specimens younger than one year were collected. More information about the method used was reported by Vlach et al. (2009b).

Data about water physical and chemical properties were repeatedly collected on the streams mentioned above. In each site $\mathrm{pH}$, conductivity, dissolved oxygen, BOD5 (the amount of dissolved oxygen consumed in five days by biological processes breaking down organic matter), undissolved particles, ammonium $\left(\mathrm{NH}_{3}\right)$, ammonium ion $\left(\mathrm{NH}_{4}^{+}\right)$, nitrites $\left(\mathrm{NO}_{2}^{-}\right)$, nitrates $\left(\mathrm{NO}_{3}^{-}\right)$, phosphorus $(\mathrm{P})$, calcium $\left(\mathrm{Ca}^{2+}\right)$ and sulphates $\left(\mathrm{SO}_{4}^{2-}\right)$ were examined. The sampling campaigns were carried out during 2007-2011. Water quality sampling was conducted once a 
month using standard methods and equipment (ISO EN standard norm protocols). Samples of water were taken in 2-litre plastic bottles. Analysis was done by a certified laboratory using standard methods.

To evaluate the relationship between water parameters and population density, only the relevant data were chosen, i.e. the dataset of 124 lines consisting of simultaneously taken data about water quality and population density of crayfish.

\section{> STATISTICAL ANALYSES}

One-way analysis of variance (ANOVA) was used for the abundance of stone crayfish and each physical and chemical parameter of water to find significant differences among sites. After this analysis, the Tukey-Kramer Test was carried out to find individual differences among sites.

In order to reveal possible correlations among the examined water parameters, a correlation matrix was created. Redundancy analysis (RDA) was undertaken to explain and better illustrate the differences/similarities over the sampling sites. The eigenvalues and the percentage explained by all the axes were estimated. The significance of the model thus created was tested using the Monte-Carlo randomisation test on the first axis and all the axes of eigenvalue and the trace (i.e. the sum of all the canonical eigenvalues) with 499 permutations, the number sufficient for the evaluation of the model at a significance level of $0.2 \%(P<0.002)$. In order to find the relationships between the abundance of stone crayfish and the water parameters, models taking into account all or some of the parameters were tested using polynomial regression. The significance of the coefficient of determination for all models using the $F$-test, as well as the importance of individual parameters using the $t$-test, was tested. In an attempt to find some gradients in abundances of localities grouped along the first axis of the above-mentioned RDA model, linear regression, the Pearson correlation coefficient and the $t$-test (evaluation of the significance of the slope) were used.

All these analyses (except for RDA) were carried out at a significance level of $5 \%$ and were evaluated using the NCSS software package. RDA was carried out in CANOCO 4.5 software and the biplot outputs were displayed using the graphic module CanoDraw.

\section{RESULTS}

The population density of stone crayfish varied in the examined streams between 0.01$5.01 \mathrm{spms} \cdot \mathrm{m}^{-2}$. Significant differences among sites were proven (ANOVA, $F=3.44, P<$ 0.001). The observed population densities are depicted as box plots in Figure 2.

The relations among the observed parameters are clear from the correlation matrix depicted in Table I. A lot of parameters were significantly correlated (Pearson Correlation Coefficient, $R^{2}<0.31$ ).

The descriptive statistics of the observed physical and chemical water characteristics (mean, 95\% confidence interval) obtained from the analyses are shown in Table lla.

A one-way ANOVA test applied for physical and chemical water properties revealed significant differences among sites in all the evaluated characteristics $(\mathrm{pH}-$ conductivity $-F=110.04$, $P=0.000$, dissolved oxygen $F=1.72, P=0.007, \mathrm{BSK}_{5}-F=2.04, P<0.001$, undissolved particles $-F=2.06, P<0.001, \mathrm{NH}_{3}-F=1.95, P=0.001, \mathrm{NH}_{4}^{+}-F=4.44, P<0.001, \mathrm{NO}_{2}^{-}-$ $F=4.44, P<0.001, \mathrm{NO}_{3}^{-}-F=21.66, P<0.001$, phosphorus $F=14.19, P<0.001, \mathrm{Ca}^{2+}-$ $F=109.53, P<0.001, \mathrm{SO}_{4}^{2}-F=37.7, P<0.001$ ).

When focused on the individual significant differences after the application of the TukeyKramer Post-Hoc Test (at a significance level of 0.05), it is possible to claim that there is a higher amount of dissolved oxygen in the Stroupínský brook than in the Mešenský brook or the Zlatý brook $(D F=245$, MSE $=3.268$, critical value $=5.586)$, a higher level of BOD5 in the Huníkovský brook than in the majority of other sites $(D F=242, M S E=5.923$, critical 


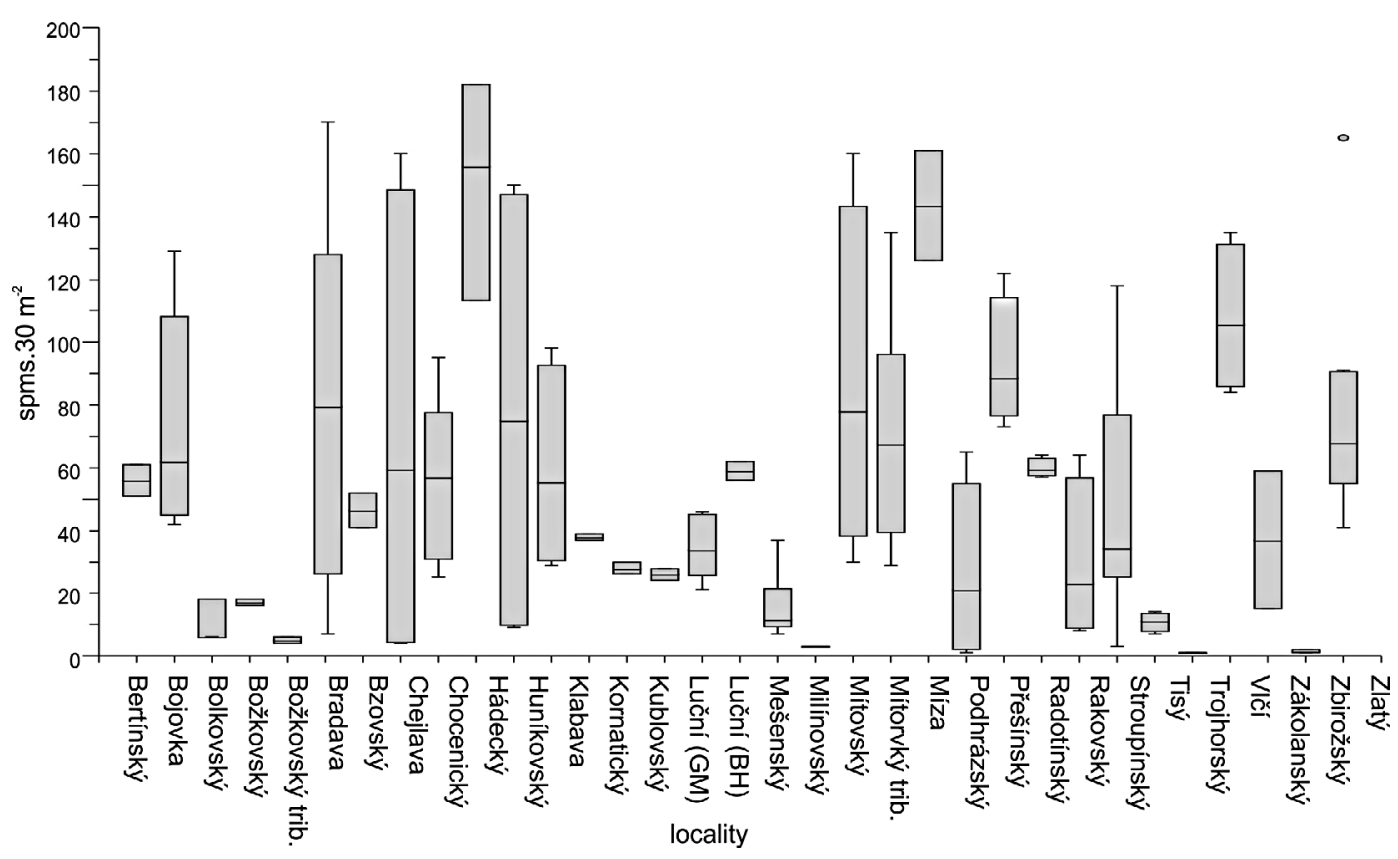

\section{Figure 2}

Population densities of the stone crayfish Austropotamobius torrentium (median, interquartile distances, confidence interval of $95 \%$ and extremes) in each examined stream. The horizontal axis shows the number of specimens of stone crayfish caught at each sample site $\left(30 \mathrm{~m}^{2}\right)$.

\section{Table I}

Correlation matrix among observed chemical and physical characteristics of water (cond - conductivity, oxygen - dissolved oxygen, UP - undissolved particles).

\begin{tabular}{|l|c|c|c|c|c|c|c|c|c|c|c|c|c|}
\cline { 2 - 16 } \multicolumn{1}{c|}{} & $\mathrm{pH}$ & cond & oxygen & $\mathrm{BOD} 5$ & $\mathrm{UP}$ & $\mathrm{NH}_{3}$ & $\mathrm{NH}_{4}^{+}$ & $\mathrm{NO}_{2}^{-}$ & $\mathrm{NO}_{3}^{-}$ & $\mathbf{P}$ & $\mathrm{Cl}^{-}$ & $\mathrm{Ca}^{2+}$ & $\mathrm{SO}_{4}^{2-}$ \\
\hline $\mathbf{p H}$ & $\times$ & 0.40 & 0.36 & -0.13 & -0.11 & 0.07 & 0.05 & 0.06 & 0.18 & 0.02 & 0.19 & 0.43 & 0.38 \\
\hline cond & 0.40 & $\times$ & 0.09 & 0.09 & 0.04 & 0.11 & 0.43 & 0.45 & 0.58 & 0.48 & 0.82 & 0.95 & 0.78 \\
\hline oxygen & 0.36 & 0.09 & $\times$ & -0.22 & -0.08 & -0.08 & -0.38 & -0.28 & 0.23 & -0.21 & 0.07 & 0.04 & 0.12 \\
\hline $\mathbf{B S K}_{5}$ & -0.13 & 0.09 & -0.22 & $\times$ & 0.30 & -0.04 & 0.25 & 0.22 & 0.01 & 0.34 & 0.15 & 0.09 & -0.04 \\
\hline $\mathbf{U P}$ & -0.11 & 0.04 & -0.08 & 0.30 & $\times$ & 0.50 & 0.19 & 0.11 & 0.11 & 0.13 & 0.11 & 0.07 & 0.09 \\
\hline $\mathbf{N H}_{3}$ & 0.07 & 0.11 & -0.08 & -0.04 & 0.50 & $\times$ & 0.34 & 0.28 & 0.08 & 0.16 & 0.06 & 0.17 & 0.13 \\
\hline $\mathbf{N H}_{4}^{+}$ & 0.05 & 0.43 & -0.38 & 0.25 & 0.19 & 0.34 & $\times$ & 0.81 & 0.28 & 0.54 & 0.42 & 0.52 & 0.31 \\
\hline $\mathbf{N O}_{2}^{-}$ & 0.06 & 0.45 & -0.28 & 0.22 & 0.11 & 0.28 & 0.81 & $\times$ & 0.46 & 0.73 & 0.50 & 0.50 & 0.29 \\
\hline $\mathbf{N O}_{3}^{-}$ & 0.18 & 0.58 & 0.23 & 0.01 & 0.11 & 0.08 & 0.28 & 0.46 & $\times$ & 0.59 & 0.60 & 0.54 & 0.32 \\
\hline $\mathbf{P}$ & 0.02 & 0.48 & -0.21 & 0.34 & 0.13 & 0.16 & 0.54 & 0.73 & 0.59 & $\times$ & 0.60 & 0.45 & 0.12 \\
\hline $\mathbf{C l}^{-}$ & 0.19 & 0.82 & 0.07 & 0.15 & 0.11 & 0.06 & 0.42 & 0.50 & 0.60 & 0.60 & $\times$ & 0.68 & 0.53 \\
\hline $\mathbf{C a}^{2+}$ & 0.43 & 0.95 & 0.04 & 0.09 & 0.07 & 0.17 & 0.52 & 0.50 & 0.54 & 0.45 & 0.68 & $\times$ & 0.78 \\
\hline $\mathbf{S O}_{4}^{2-}$ & 0.38 & 0.78 & 0.12 & -0.04 & 0.09 & 0.13 & 0.31 & 0.29 & 0.32 & 0.12 & 0.53 & 0.78 & $\times$ \\
\hline
\end{tabular}

value $=5.587$ ), a higher level of undissolved particles in the Chocenický, Zákolanský and Luční brooks (Central Bohemia Highlands) $(D F=244$, MSE $=356.629$, critical value $=5.587$ ), a higher amount of free ammoniac in the Huníkovský brook ( $D F=241$, MSE $=4.267$, critical value $=5.588$ ), a higher level of ammonium ion in the Zákolanský brook than in other sites $(D F=252, \mathrm{MSE}=2.902$, critical value $=5.584)$ and a higher level of nitrites in the Radotínský and Zákolanský brooks $(D F=251, \mathrm{MSE}=1.386$, critical value $=5.585)$.

Redundancy analysis (RDA) was used to find some similarities/differences among sites based on the configuration of physical and chemical properties of water. The chosen model explains $10.5 \%$ of sites' variability and $57.5 \%$ of sites' water parameter relations (Monte-Carlo Permutation Test, $F=2.611, P<0.002$ ). The biplot of the first two axes is depicted in Figure 3 . In order to find a possible relationship between the gradient of localities along the 1st axis 
Table lla

Basic statistics for the observed physical and chemical parameters of water $(\bar{X}-$ mean, $\mathrm{Cl}-95 \%$ confidence interval). $b$ - brook, LT - left tributary, BH - the Bohemian Highlands, GM - the Giant Mountains.

\begin{tabular}{|c|c|c|c|c|c|c|c|c|c|c|c|c|}
\hline \multirow[b]{2}{*}{ Locality } & \multicolumn{2}{|r|}{ pH } & \multicolumn{2}{|c|}{$\begin{array}{l}\text { Conductivity } \\
\left(\text { (nS. } \mathrm{cm}^{-1}\right)\end{array}$} & \multicolumn{2}{|c|}{$\begin{array}{c}\mathrm{O}^{2} \\
\left(\mathrm{mg} \cdot \mathrm{L}^{-1}\right)\end{array}$} & \multicolumn{2}{|r|}{$\begin{array}{c}\mathrm{BSK}_{5} \\
\left(\mathrm{mg} \cdot \mathrm{L}^{-1}\right)\end{array}$} & \multicolumn{2}{|c|}{$\begin{array}{l}\text { Undisolved } \\
\left(\mathrm{mg} \cdot \mathrm{L}^{-1}\right)\end{array}$} & \multicolumn{2}{|r|}{$\begin{array}{c}\mathrm{NH}_{3} \\
\left(\mathrm{mg} \cdot \mathrm{L}^{-1}\right)\end{array}$} \\
\hline & $\bar{x}$ & $C /(\alpha=0.95)$ & $\bar{x}$ & $C I(\alpha=0.95)$ & $\bar{x}$ & $C /(\alpha=0.95)$ & $\bar{x}$ & $C /(\alpha=0.95)$ & $\bar{x}$ & $C /(\alpha=0.95)$ & $\bar{x}$ & $C I(\alpha=0.95)$ \\
\hline Bertínský b. & 8.41 & $8.40-8.41$ & 518 & $518-518$ & 11.9 & $11.9-11.9$ & 1.5 & $1.5-1.5$ & 1.6 & $1.6-1.6$ & 0.0007 & $0.0006-0.0007$ \\
\hline Bojovka 1 & 7.82 & $7.66-7.99$ & 278 & 265-292 & 8.97 & $8.37-9.57$ & 1.47 & $0.89-2.1$ & 6.79 & $2.43-11.1$ & 0.0008 & $0.0004-0.0012$ \\
\hline Bojovka 2 & 7.70 & $7.70-7.70$ & 227 & $191-264$ & 9.2 & $8.51-9.89$ & 2.4 & $0.18-4.6$ & 4.9 & $3.1-6.7$ & 0.0005 & $0.0004-0.0005$ \\
\hline Bolkovský b. & 7.92 & $7.69-8.15$ & 322 & 280-364 & 9.09 & $8.65-9.53$ & 1.31 & $0.44-2.2$ & 5.27 & $3.14-7.39$ & 0.0024 & $0.0005-0.0043$ \\
\hline Božkovský b. & 8.07 & $8.04-8.10$ & 1040 & $883-1197$ & 10.33 & $9.57-11.08$ & 1.85 & $1.7-2$ & 23.2 & | 14.2-32.2 & 0.0012 & $0.0008-0.0016$ \\
\hline Božkovský b. LT & 8.07 & $8.07-8.07$ & 1180 & 1150-1209 & 10.42 & $9.48-11.36$ & 1.55 & $1.06-2$ & 5.6 & $2.83-8.37$ & 0.0007 & $0.0006-0.0008$ \\
\hline Bradava 2 & 7.53 & $7.35-7.70$ & 247 & $233-260$ & 8.48 & $7.59-9.37$ & 2.08 & $1.55-2.6$ & 5.03 & $2.48-7.57$ & 0.0009 & $0.0005-0.0013$ \\
\hline Bradava 3 & 7.63 & $7.42-7.85$ & 192 & $160-224$ & 9.8 & $9.25-10.35$ & 1.17 & $0.79-1.5$ & 7.73 & $1.97-13.5$ & 0.0005 & $0.0003-0.0007$ \\
\hline Bradava 4 & 7.51 & $7.51-7.51$ & 158 & $148-168$ & 10.04 & $7.32-12.76$ & 2.45 & $1.69-3.2$ & 2.4 & $2.4-2.4$ & 0.0009 & $0.0000-0.0019$ \\
\hline Bradava 5 & 7.78 & $7.70-7.86$ & 177 & $173-181$ & 9.2 & $8.01-10.4$ & 1.5 & $0.96-2.4$ & 8.33 & $2.76-13.9$ & 0.0005 & $0.0004-0.0005$ \\
\hline Bzovský b. & 7.75 & $7.68-7.82$ & 514 & $486-542$ & 9.91 & $9.38-10.44$ & 1.54 & $1.18-1.9$ & 14.1 & $6.14-22.1$ & 0.0005 & $0.0005-0.0017$ \\
\hline Hrádecký b. & 7.57 & $7.46-7.57$ & 255 & $195-315$ & 9.2 & $7.75-10.65$ & 1.8 & $0.98-2.6$ & 2.6 & $1.02-4.18$ & 0.0003 & $0.0003-0.0004$ \\
\hline Huníkovský b. & 7.62 & $7.48-7.76$ & 256 & $204-308$ & 9.69 & $8.62-10.75$ & 8.05 & $0.45-16$ & 28.9 & $3.66-54$ & 0.0166 & $0.0000-0.0358$ \\
\hline Hûrecký b. & 7.58 & $7.40-7.77$ & 162 & $156-168$ & 9.91 & $9.03-10.79$ & 1.4 & $1.15-1.7$ & 5.24 & $3.36-7.12$ & 0.0007 & $0.0005-0.0008$ \\
\hline Chejlava & 7.57 & $7.53-7.62$ & 501 & $471-531$ & 9.86 & $9.15-10.58$ & 1.83 & $1.63-2$ & 13.2 & $11.9-14.5$ & 0.0002 & $0.0002-0.0002$ \\
\hline Chocenický b. & 7.73 & $7.65-7.81$ & 314 & $307-321$ & 8.84 & $8.26-9.41$ & 4.8 & $3.42-6.2$ & 39.5 & $16.9-62.1$ & 0.0012 & $0.0006-0.0017$ \\
\hline Klabava & 7.49 & 7.32-7.65 & 94.8 & $75.9-114$ & 10.06 & $9.26-10.86$ & 2.92 & $1.49-4.4$ & 18 & $0.00-36.1$ & 0.0012 & $0.0001-0.0024$ \\
\hline Kornatický b. & 7.69 & $7.63-7.75$ & 358 & $328-388$ & 9.24 & $8.13-10.35$ & 2.14 & $1.78-2.5$ & 19.6 & $0.00-40.5$ & 0.0010 & $0.0007-0.0013$ \\
\hline Kublovský b. & 7.89 & 7.79-7.99 & 497 & $480-514$ & 10.08 & $9.4-10.75$ & 1.62 & $0.63-2.6$ & 4.58 & $1.35-7.81$ & 0.0007 & $0.0005-0.0009$ \\
\hline Luční b. (BH) & 7.87 & $7.73-8.01$ & 325 & $320-330$ & 9.11 & $8.77-9.45$ & 1.69 & $0.89-2.5$ & 5.87 & $2.94-8.79$ & 0.0019 & $0.0080-0.0030$ \\
\hline Luční b. (GM) & 7.75 & $7.65-7.86$ & 534 & $477-591$ & 9.26 & $8.12-10.39$ & 1.26 & $1.1-1.4$ & 45.2 & $9.32-81.1$ & 0.0008 & $0.0004-0.0012$ \\
\hline Mešenský b. & 7.28 & $7.20-7.37$ & 314 & $292-336$ & 7.8 & $7.6-7.99$ & 1.8 & $1.12-2.5$ & 3.33 & $2.46-4.2$ & 0.0003 & $0.0002-0.0005$ \\
\hline Milínovský b. & 7.65 & $7.65-7.65$ & 166 & $144-188$ & 10.37 & $9.17-11.56$ & 1.25 & $0.76-1.7$ & 11 & $10.9-11$ & 0.0004 & $0.0001-0.0006$ \\
\hline Mítovský b. & 7.51 & $7.44-7.58$ & 226 & $222-229$ & 10.01 & $8.64-11.37$ & 1.53 & $0.92-2.1$ & 4.47 & $3.08-5.85$ & 0.0002 & $0.0001-0.0003$ \\
\hline Mítovský b. LT & 7.58 & $7.36-7.80$ & 166 & $153-179$ & 9.73 & $8.9-10.56$ & 1.23 & $0.99-1.5$ & 12.4 & $3.55-21.3$ & 0.0004 & $0.0001-0.0006$ \\
\hline Míza & 7.61 & $7.07-8.14$ & 390 & $340-440$ & 7.79 & $4.65-10.92$ & 1.65 & $0.75-2.6$ & 4.8 & $0.92-8.68$ & 0.0005 & $0.0002-0.0007$ \\
\hline Podhrázský p. & 7.61 & $7.45-7.76$ & 331 & $321-342$ & 7.87 & $6.85-8.89$ & 1.75 & $1.19-2.3$ & 6.9 & $2.65-11.2$ & 0.0007 & $0.0003-0.0010$ \\
\hline Přešínský b. & 7.65 & $7.51-7.80$ & 339 & 293-385 & 9.27 & $8.33-10.2$ & 1.57 & $1.13-2$ & 6.42 & $0.29-12.6$ & 0.0006 & $0.0002-0.0010$ \\
\hline Radotínský b. & 8.04 & $7.81-8.27$ & 1065 & $1033-1096$ & 9.53 & $8.55-10.52$ & 2.77 & $2.19-3.4$ & 12.8 & $7.70-17.9$ & 0.0119 & $0.0003-0.0234$ \\
\hline Rakovský b. & 7.49 & $7.35-7.63$ & 325 & $315-335$ & 8.98 & 7.73-10.22 & 2.28 & $1.45-3.1$ & 10.3 & $4.55-16$ & 0.0006 & $\mid 0.0002-0.0010$ \\
\hline Skořický b. & 7.22 & $7.22-7.22$ & 133 & $81.7-184$ & 10.54 & $8.93-12.15$ & 1.55 & $1.34-1.8$ & 2.85 & $0.29-5.41$ & 0.0001 & $0.0001-0.0001$ \\
\hline Stroupínský b. 1 & 8.10 & $7.91-8.29$ & 689 & $662-716$ & 11.12 & $9.85-12.39$ & 2.39 & $1.76-3$ & 6.51 & $4.90-8.13$ & 0.0029 & $0.0016-0.0042$ \\
\hline Stroupínský b. 2 & 8.03 & $7.89-8.16$ & 714 & $680-749$ & 10.49 & $9.63-11.36$ & 2.34 & $1.68-3$ & 8.86 & $6.64-1.08$ & 0.0026 & $0.0012-0.0039$ \\
\hline Stroupínský b. 3 & 7.92 & $7.68-8.16$ & 695 & $644-748$ & 9.63 & $8.25-11.01$ & 3.53 & $2.07-5$ & 9.86 & $7.41-12.3$ & 0.0009 & $0.0004-0.0013$ \\
\hline Tisý b. & 6.96 & $6.65-7.27$ & 192 & $160-224$ & 7.47 & $6.76-8.18$ & 4.05 & $2.42-5.7$ & 24.4 & $12.1-36.6$ & 0.0005 & $0.0002-0.0008$ \\
\hline Trojhorský b. LT & 8.00 & $7.86-8.14$ & 743 & $674-811$ & 9.69 & $8.77-10.61$ & 1.81 & $1.04-2.6$ & 13 & $8.68-17.3$ & 0.0055 & $0.0004-0.0107$ \\
\hline Vlčí b. & 7.94 & $7.9-8$ & 396 & $371-422$ & 10.61 & $10.1-11.09$ & 3.36 & $3.11-3.6$ & 21 & $16.1-25.9$ & 0.0012 & $0.0008-0.002$ \\
\hline Zákolanský b. & 7.94 & $7.78-8.1$ & 1038 & $1014-1063$ & 9.12 & $7.4-10.83$ & 4.14 & $3.04-5.2$ & 44.5 & $21.7-67.3$ & 0.0091 & $0.0016-0.017$ \\
\hline Zbirožský b. & 8.01 & $7.91-8.1$ & 429 & $406-452$ & 10.56 & $9.06-12.05$ & 1.92 & $0.98-2.9$ & 12.5 & $0.98-2.86$ & 0.0007 & $0.0003-0.001$ \\
\hline Zlatý b. & 7.6 & $7.47-7.7$ & 389 & $349-429$ & 8 & $7.2-8.8$ & 2.35 & $1.65-3.1$ & 15.8 & $9-22.6$ & 0.0008 & $0.0006-0.001$ \\
\hline
\end{tabular}




\section{Table Ilb}

Basic statistics for the observed physical and chemical parameters of water (mean and 95\% confidence interval). $L T$ - left tributary, BH - the Bohemian Highlands, GM - the Giant Mountains.

\begin{tabular}{|c|c|c|c|c|c|c|c|c|c|c|c|c|}
\hline \multirow[b]{2}{*}{ Locality } & \multicolumn{2}{|r|}{$\begin{array}{c}\mathrm{NH}_{4}^{+} \\
\left(\mathbf{m g} \cdot \mathbf{L}^{-1}\right)\end{array}$} & \multicolumn{2}{|r|}{$\begin{array}{c}\mathrm{NO}_{2}^{2-} \\
\left(\mathrm{mg} \cdot \mathrm{L}^{-1}\right)\end{array}$} & \multicolumn{2}{|r|}{$\begin{array}{c}\mathrm{NO}_{3}^{2-} \\
\left(\mathrm{mg} \cdot \mathrm{L}^{-1}\right)\end{array}$} & \multicolumn{2}{|r|}{$\begin{array}{c}P \\
\left(\mathrm{mg} \cdot \mathrm{L}^{-1}\right)\end{array}$} & \multicolumn{2}{|r|}{$\begin{array}{c}\mathrm{Ca} \\
\left(\mathrm{mg} \cdot \mathrm{L}^{-1}\right)\end{array}$} & \multicolumn{2}{|c|}{$\begin{array}{c}\mathrm{SO}_{4}^{2-} \\
\left(\mathrm{mg} \cdot \mathrm{L}^{-1}\right)\end{array}$} \\
\hline & $\bar{x}$ & $C /(\alpha=0.95)$ & $\bar{x}$ & $C /(\alpha=0.95)$ & $\bar{x}$ & $C /(\alpha=0.95)$ & $\bar{x}$ & $C /(\alpha=0.95)$ & $\bar{x}$ & $\operatorname{cl}(\alpha=0.95)$ & $\bar{x}$ & $C I(\alpha=0.95)$ \\
\hline Bertínský b. & 0.02 & $0.02-0$ & 0 & $0.00-0.00$ & 8.28 & $8.28-8.28$ & 0.02 & $0.02-0.02$ & 71.9 & $71.9-71.9$ & 83.1 & $83.1-83.1$ \\
\hline Bojovka 1 & 0.04 & $0.03-0.1$ & 0.02 & $0.00-0.04$ & 8.58 & $3.99-13.17$ & 0.05 & 0.030 .06 & 30.7 & $28.0-33.5$ & 30.0 & $27.18-32.82$ \\
\hline Bojovka 2 & 0.03 & $0.03-0$ & 0.01 & $0.01-0.02$ & 1.21 & $1.11-1.31$ & 0.03 & $0.02-0.05$ & 23.4 & $22.2-24.7$ & 17.0 & $16.31-17.69$ \\
\hline Bolkovský b. & 0.1 & $0.06-0.1$ & 0.1 & $0.02-0.19$ & 14.9 & $12.71-17.27$ & 0.15 & $0.09-0.22$ & 45.6 & $38.0-53.1$ & 31.9 & $25.0-38.3$ \\
\hline Božkovský b. & 0.05 & $0.04-0.1$ & 0.03 & $0.02-0.05$ & 33.79 & $29.89-38.60$ & 0.03 & $0.01-0.05$ & 139 & $116-16.6$ & 198 & $113.7-282.3$ \\
\hline Božkovský b. LT & 0.03 & $0.03-0$ & 0.01 & $0.00-0.01$ & 2.1 & $0.00-4.72$ & 0.02 & $0.01-0.04$ & 125 & $124-126$ & 273 & $237.0-309.0$ \\
\hline Bradava 2 & 0.09 & $0.05-0.1$ & 0.12 & $0.04-0.20$ & 4.23 & $3.00-5.45$ & 0.12 & $0.08-0.16$ & 24.8 & $23.0-26.6$ & 27.2 & $23.18-31.22$ \\
\hline Bradava 3 & 0.05 & $0.02-0.1$ & 0.03 & $0.01-0.04$ & 2.31 & $1.27-3.36$ & 0.04 & $0.04-0.05$ & 18.2 & $14.2-22.1$ & 27.4 & $19.7-35.0$ \\
\hline Bradava 4 & 0.09 & $0.05-0.1$ & 0.02 & $0.00-0.03$ & 3.27 & $0.25-6.30$ & 0.03 & $0.02-0.03$ & 15.2 & $14.7-15.6$ & 24.2 & $24.2-24.2$ \\
\hline Bradava 5 & 0.04 & $0.02-0.1$ & 0.02 & $0.01-0.04$ & 2.53 & $0.78-4.28$ & 0.04 & $0.03-0.05$ & 15.7 & $13.9-17.4$ & 15.3 & $13.86-16.74$ \\
\hline Bzovský b. & 0.08 & $0.03-0$ & 0.03 & $0.02-0.04$ & 17.48 & $16.08-18.88$ & 0.07 & $0.06-0.09$ & 51.8 & $67.5-55.5$ & 71.0 & $67.46-74.55$ \\
\hline Hrádecký b. & 0.04 & $0.04-0.1$ & 0 & $0.00-0.00$ & 2.25 & $0.00-4.98$ & 0.02 & $0.01-0.03$ & 29.9 & $21.4-38.4$ & 22.6 & $20.38-24.82$ \\
\hline Huníkovský b. & 0.06 & $0.05-0.1$ & 0.02 & $0.02-0.03$ & 3.59 & $3.06-4.12$ & 0.22 & $0.03-0.42$ & 25.5 & $22.3-28.8$ & 38.3 & $29.09-47.51$ \\
\hline Hûrecký b. & 0.08 & $0.06-0.1$ & 0.03 & $0.01-0.05$ & 5.47 & $5.17-5.76$ & 0.07 & $0.05-0.09$ & 17.1 & $16.3-17.9$ & 15.01 & $12.32-17.71$ \\
\hline Chejlava & 0.03 & $0.03-0$ & 0.02 & $0.01-0.03$ & 17.32 & $8.37-26.27$ & 0.16 & $0.15-0.17$ & 38.1 & $36.7-39.5$ & 41.17 & $33.52-48.81$ \\
\hline Chocenický b. & 0.07 & $0.03-0.1$ & 0.06 & $0.04-0.07$ & 5.06 & $3.76-6.35$ & 0.29 & $0.17-0.40$ & 34.2 & $33.0-35.4$ & 28.65 & $27.96-29.33$ \\
\hline Klabava & 0.29 & $0.00-0.60$ & 0.04 & $0.00-0.07$ & 2.61 & $1.11-4.10$ & 0.1 & $0.04-0.15$ & 9.68 & $6.6-12.8$ & 9.18 & $6.91-11.45$ \\
\hline Kornatický b. & 0.09 & $0.07-0.10$ & 0.09 & $0.05-0.12$ & 10.32 & $7.44-13.20$ & 0.09 & $0.04-0.14$ & 41.1 & $35.7-46.5$ & 39.43 & $34.84-44.03$ \\
\hline Kublovský b. & 0.04 & $0.03-0.05$ & 0.01 & $0.01-0.01$ & 10.23 & $9.08-11.37$ & 0.05 & $0.03-0.06$ & 51.7 & $48.7-54.7$ & 55.65 & $50.45-60.84$ \\
\hline Luční b. (BH) & 0.05 & $0.04-0.06$ & 0.01 & $0.01-0.02$ & 16.44 & $11.03-21.85$ & 0.12 & $0.06-0.17$ & 68.7 & $61.1-76.3$ & 105.05 & $91.26-118.8$ \\
\hline Luční b. (GM) & 0.1 & $0.03-0.18$ & 0.06 & $0.04-0.08$ & 8.54 & $7.84-9.25$ & 0.12 & $0.10-0.15$ & 44.6 & $43.3-45.9$ & 36.05 & $34.24-37.86$ \\
\hline Mešenský b. & 0.06 & $0.04-0.08$ & 0.06 & $0.02-0.11$ & 11.14 & $8.62-13.67$ & 0.27 & $0.20-0.35$ & 28.9 & $27.6-30.1$ & 47.05 & $44.36-49.74$ \\
\hline Milínovský b. & 0.04 & $0.02-0.1$ & 0.02 & $0.01-0.02$ & 4.09 & $0.00-8.43$ & 0.02 & $0.00-0.04$ & 26.9 & $19.4-34.3$ & 19.0 & $13.87-24.13$ \\
\hline Mítovský b. & 0.02 & $0.02-0.03$ & 0.02 & $0.01-0.02$ & 4.14 & $0.84-7.43$ & 0.03 & $0.02-0.04$ & 21.7 & $20.1-23.3$ & 36.03 & $31.29-40.78$ \\
\hline Mítovský b. LT & 0.03 & $0.01-0.04$ & 0.02 & $0.01-0.03$ & 3.68 & $2.54-4.82$ & 0.02 & $0.02-0.03$ & 13.9 & $12.5-15.4$ & 11.45 & $9.3-13.61$ \\
\hline Míza & 0.05 & $0.04-0.07$ & 0 & $0.00-0.00$ & 1.09 & $0.41-1.78$ & 0.02 & $0.01-0.03$ & 43.6 & $36.5-50.7$ & 55.84 & 50.39-61.29 \\
\hline Podhrázský p. & 0.06 & $0.04-0.07$ & 0.01 & $0.00-0.02$ & 1.59 & $0.66-2.51$ & 0.04 & $0.02-0.05$ & 38.9 & $36.3-41.5$ & 14.6 & $13.39-15.81$ \\
\hline Přešínský b. & 0.04 & $0.03-0.06$ & 0.01 & $0.01-0.02$ & 6 & $4.36-7.64$ & 0.05 & $0.02-0.07$ & 36.5 & $30.0-43.0$ & 49.64 & $42.62-56.66$ \\
\hline Radotínský b. & 0.34 & $0.13-0.55$ & 0.46 & $0.18-0.75$ & 39.95 & $34.88-45.02$ & 0.83 & $0.64-1.03$ & 146 & $142-158$ & 154.28 & 145.3-163.3 \\
\hline Rakovský b. & 0.06 & $0.05-0.07$ & 0.06 & $0.00-0.12$ & 4.13 & $2.51-5.75$ & 0.04 & $0.03-0.06$ & 33 & 33.034 .7 & 29.7 & $27.25-32.15$ \\
\hline Skořický b. & 0.03 & $0.01-0.04$ & 0.01 & $0.00-0.02$ & 4.09 & $0.63-7.55$ & 0.03 & $0.00-0.05$ & 12.4 & $6.2-18.6$ & 24.05 & $20.38-27.72$ \\
\hline Stroupínský b. 1 & 0.1 & $0.05-0.15$ & 0.08 & $0.05-0.10$ & 16.64 & $14.05-19.24$ & 0.34 & $0.27-0.41$ & 69 & $65.9-72.1$ & 85.07 & $79.5-90.63$ \\
\hline Stroupínský b. 2 & 0.12 & $0.03-0.21$ & 0.13 & $0.08-0.18$ & 22.82 & $19.81-25.82$ & 0.58 & $0.47-0.68$ & 68.6 & $65.8-71.4$ & 82 & 73.69-90.31 \\
\hline Stroupínský b. 3 & 0.05 & $0.03-0.08$ & 0.14 & $0.09-0.19$ & 27.46 & $21.03-33.89$ & 0.5 & $0.35-0.65$ & 69.3 & $65.8-72.8$ & 83.5 & $63.27-103.7$ \\
\hline Tisý b. & 0.16 & $0.12-0.21$ & 0.07 & $0.04-0.11$ & 1.81 & $0.62-3.01$ & 0.1 & $0.07-0.13$ & 26.1 & $22.1-30.2$ & 13.45 & $8.81-18.09$ \\
\hline Trojhorský b. LT & 0.15 & $0.06-0.23$ & 0.02 & $0.01-0.02$ & 7.98 & $6.25-9.70$ & 0.08 & $0.05-0.10$ & 108 & $93.7-122$ & 110.98 & $103.77-118.2$ \\
\hline Vlcí b. & 0.07 & $0.06-0.1$ & 0.11 & $0.09-0.12$ & 21.97 & $9.48-34.45$ & 0.25 & $0.20-0.30$ & 44.1 & $41.6-46.6$ & 38.4 & $29.38-47.42$ \\
\hline Zákolanský b. & 0.45 & $0.07-0.8$ & 0.36 & $0.09-0.62$ & 31.64 & $25.85-37.42$ & 0.37 & $0.24-0.50$ & 163 & $158-169$ & 152.3 & 143.2-161.4 \\
\hline Zbirožský b. & 0.03 & $0.02-0$ & 0.02 & $0.01-0.03$ & 7.11 & $3.97-10.24$ & 0.12 & $0.06-0.18$ & 47.6 & $45.4-49.9$ & 54.4 & $54.4-54.4$ \\
\hline Zlatý b. & 0.08 & $0.05-0.1$ & 0.3 & $0.2-0.40$ & 6.76 & $4.54-8.98$ & 0.08 & $0.05-0.11$ & 46.1 & $37.3-54.9$ & 27.4 & $26.22-28.58$ \\
\hline
\end{tabular}




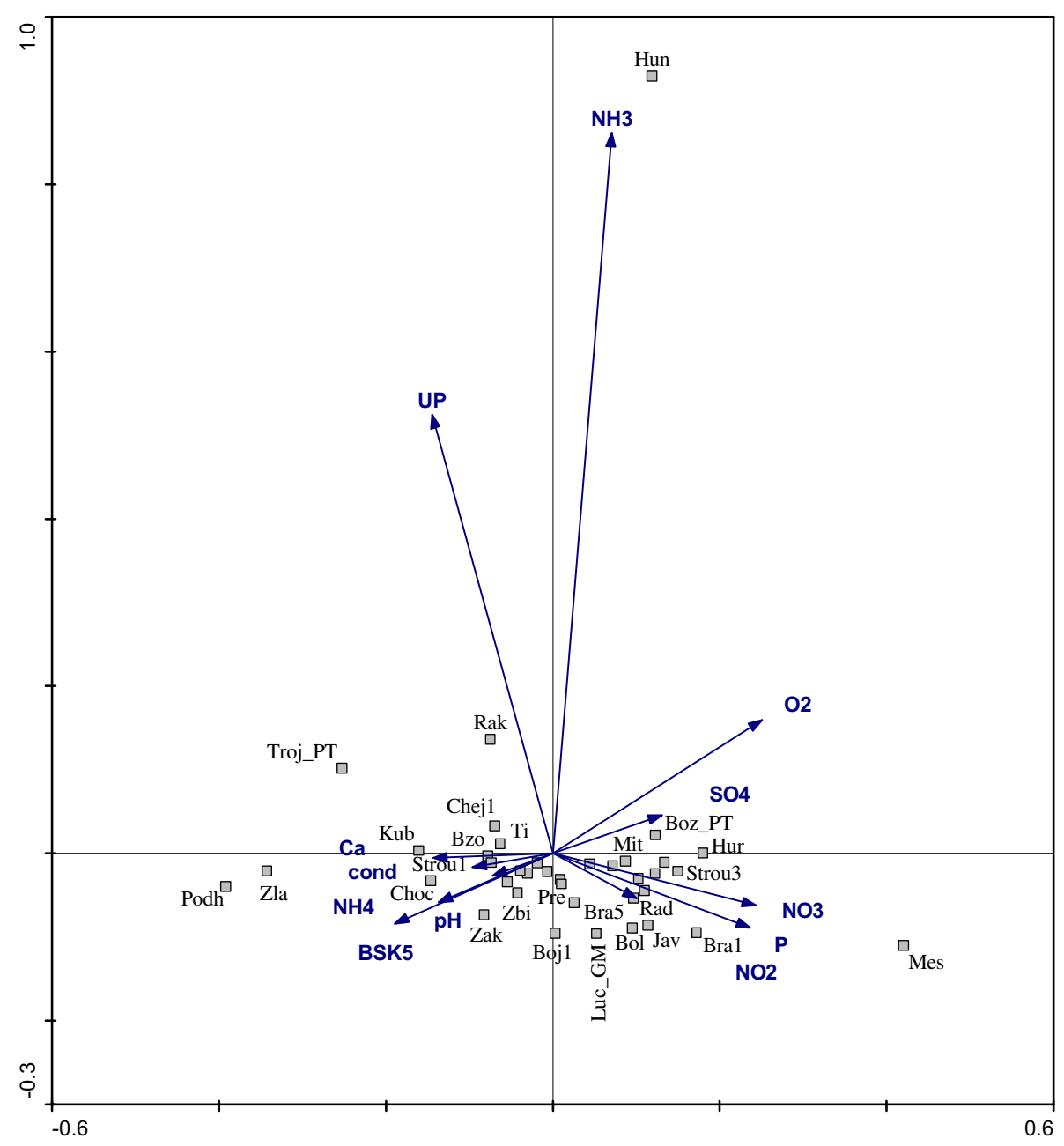

Figure 3

Differences/similarities among the study sites inhabited by the stone crayfish Austropotamobius torrentium relating observed water physical and chemical properties (RDA biplot, 1 st and 2 nd axes). For abbreviations see Table I and Figure 1.

of the RDA model and population densities, the observed abundances were plotted against the gradient of localities along the 1st axis of RDA (see Figure 4). No relationship was found $\left(R^{2}=0.004, t=-0.35, P=0.72\right)$.

The influence of water quality on population density of stone crayfish was also evaluated using polynomial regression. Firstly, all the parameters were used for the analysis. No relationship was found ( $D F=124, R^{2}=0.25, F=1.41, P=0.12$ ). However, the influence of some parameters (conductivity, nitrates, calcium and sulphates) on population density was significant $(|t|<1.97, P<0.05)$. The next model, consisting of the parameters chosen on the basis of the preliminary analysis, significantly influenced population density of stone crayfish. The relationship can be assessed as: abundance $=70.72+0.54 \times$ sulphates $0.001 \times$ sulphates $^{2}-2.43 \times$ calcium $+0.009 \times$ calcium $^{2}-3.70 \times$ nitrates $+0.10 \times$ nitrates $^{2}+0.25 \times$ conductivity $-0.002 \times$ conductivity $^{2}\left(D F=124, R^{2}=0.13, F=2.23\right.$, $P=0.02)$. The influence of each parameter on the population density is depicted in Figure 5 .

\section{DISCUSSION}

The abundance of stone crayfish in the examined localities significantly varied. The observed values correspond with other reports (Kappus et al., 1999; Renz and Breithaupt, 2000; 


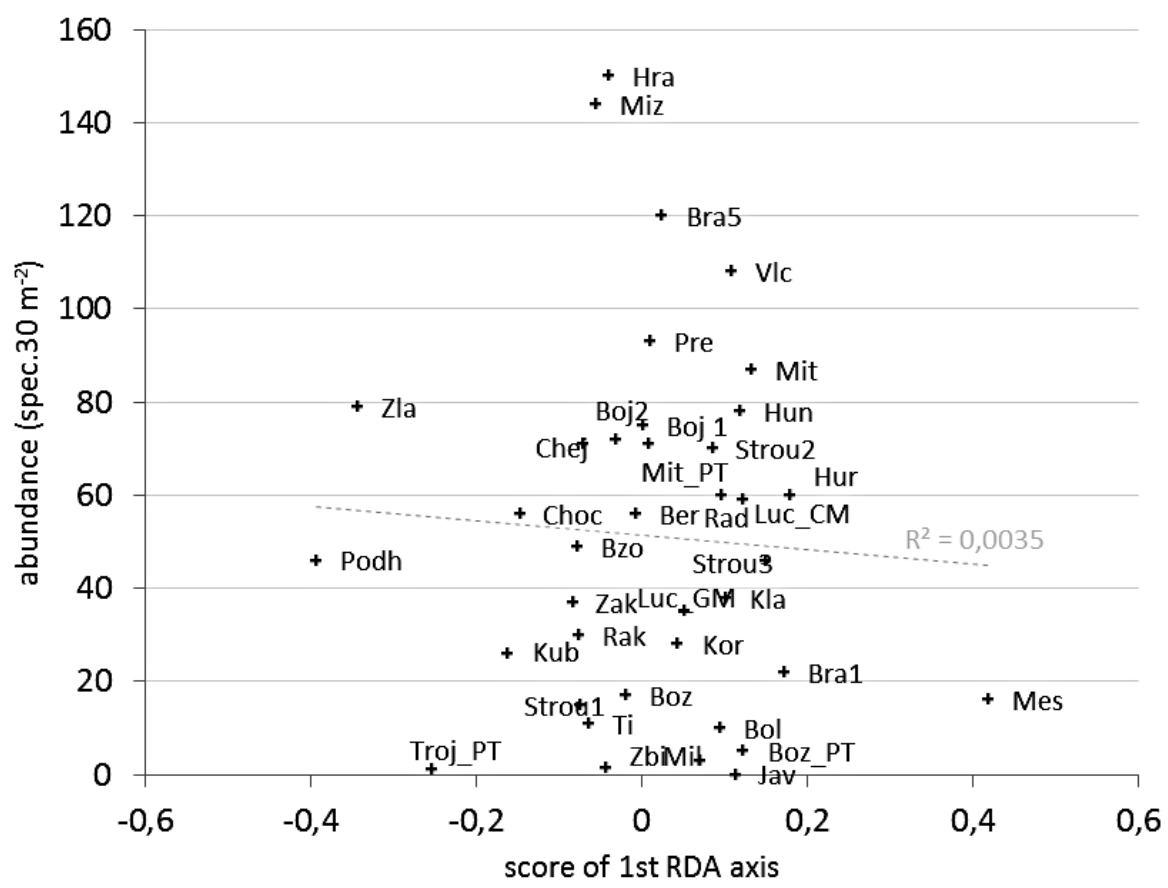

Figure 4

Abundance of the stone crayfish Austropotamobius torrentium in the observed streams grouped along the 1 st axis of RDA analysis by their differences/similarities.
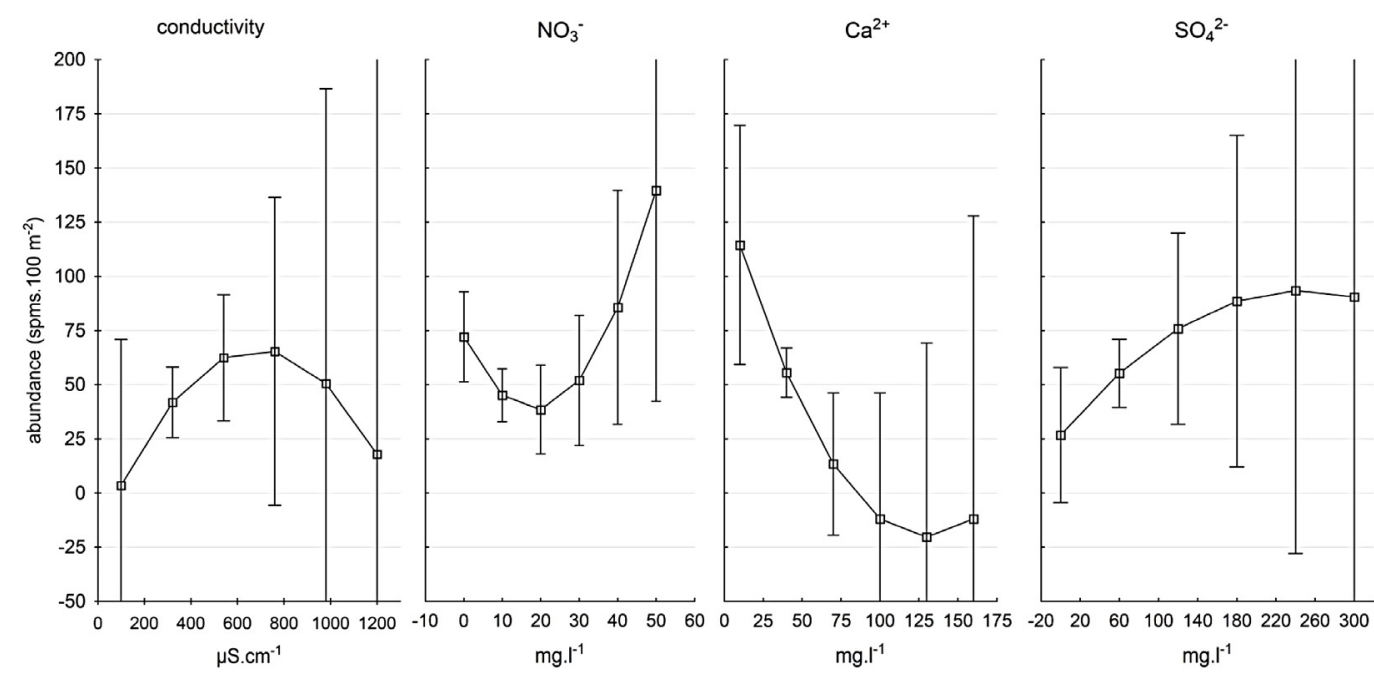

Figure 5

Relationship between the abundance of the stone crayfish Austropotamobius torrentium and conductivity, nitrates, calcium and sulphates (predicted values in the model using polynomial regression).

Štambergová et al., 2009; Vlach et al., 2009b). The aim of this study was to examine the influence of physical and chemical parameters of water on the differences in population densities. The first examined factor was $\mathrm{pH}$. There were statistical differences in the mean $\mathrm{pH}$ values at sites, which varied between 6.96 in the Tisý brook and 8.41 in the Bertínský brook. This range is slightly wider than the optimum mentioned by Renz and Breithaupt (2000), especially if the observed extremes are considered (6.31 in the Padrt'ský brook and 9.40 in the Stroupinský brook). A wide $\mathrm{pH}$ range is conditioned by the underlying geology and features of the landscape the streams flow through. The lowest $\mathrm{pH}$ was recorded in the streams which spring in 
the Brdy Mountains. This fact is connected with the acid bedrock consisting of granitites and the presence of humid acids from mosses (Tisý brook, Skořický brook, Klabava, Mítovský brook, Bradava, etc.). On the other hand, the streams with a higher level of $\mathrm{pH}$ flow through alkaline bedrocks (Bertínský brook, Radotínský brook and Zákolanský brook). The underlying geology also influences the higher amount of calcium, which was positively correlated with $\mathrm{pH}$ (see below), as pointed out by Favaro et al. (2010).

Conductivity was strongly correlated with $\mathrm{pH}$ value (see Table I). The wide range of conductivity $\left(65-1209 \mu \mathrm{S} \cdot \mathrm{cm}^{-1}\right)$ is connected to variable underlying geology, as for $\mathrm{pH}$. Kozák et al. (2002) recorded a narrower range of conductivity in the Czech Republic, but the only 4 localities known at that time were observed. The minimum was observed in the Klabava river in the Brdy Mts., and the maximum in the Božkovský brook and its tributary, which runs through the countryside in the immediate vicinity of a large deposit of clay slurry with a large amount of calcium. The relationship between conductivity and the local geology was also reported by Trouilhé et al. (2007). The mean conductivity in the waters inhabited by the stone crayfish in the Czech Republic is $422 \mu \mathrm{S} . \mathrm{cm}^{-1}$. This value is higher than the mean values mentioned by some authors (Favaro et al., 2010; Pârvulescu et al., 2011; Trouilhé et al., 2007), and lower than the value given by Broquet et al. (2002). Despite the observed correlation between the abundance and conductivity, large differences in the values of conductivity denote that this parameter is not too important for the given species. This phenomenon was also observed by Broquet et al. (2002) and Pârvulescu et al. (2011).

The amount of dissolved oxygen varied, and ranged between 7.47 and $11.9 \mathrm{mg} \cdot \mathrm{L}^{-1}$. This is consistent with data reported by Kozák et al. (2001) and Favaro et al. (2010). Hypoxia occurred in the Zákolanský brook (1.77 mg. $\left.\mathrm{L}^{-1}\right)$, also reported by Grandjean et al. (1996) and by Trouilhé et al. (2007) in the localities inhabited by A. pallipes. Although the amount of dissolved oxygen was strongly correlated with the 1st RDA axis, oxygen played no importance in the model taking into account all parameters using polynomial regression. This confirms the findings that oxygen at long-term low concentrations acts as a rather limiting factor; crayfish are fairly tolerant to decrease in water oxygen saturation and the effect of eutrophication and organic pollution, which can be characterised through the BOD5 index. We did not find any relationship between the level of organic matter and the abundance of crayfish. This finding is not surprising as higher values of BOD5 are limiting, as reported by many authors (Broquet et al., 2002; Favaro et al., 2010; Svobodová et al., 2012; Trouilhé et al., 2007). In the observed period, we recorded at least two cases of quick increase in BOD5 and rapid decrease in oxygen, which led to overall mortality of stone crayfish connected with inappropriate farming. It was in the Chocenický brook in 2007 and also in the Vlčí brook in 2012 where the values of BOD5 increased four times from January 2012 and the population was completely destroyed (Vlach, own data). Nevertheless, it is typical of many streams in the Czech Republic that the mild nutrient enrichment connected with the first village occurring on the stream enables the presence of the stone crayfish or increases its abundance. The suitability of mild nutrient enrichment for crayfish was also reported by Foster (1995) and Svobodová et al. (2012).

Another observed parameter was ammonium ion. The observed mean values of ammonium ion (see Table Ila) are comparable with data pointed out by Pârvulescu et al. (2011) and the list given by Trouilhé et al. (2007) for $A$. pallipes. They slightly extend the optimum reported by Svobodová et al. (2008). The wide range of $\mathrm{NH}_{4}$ observed in the sampling sites is related to seasonal variations in leaf canopy cover at sites located in forests, where values of ammonium ion rapidly increased in autumn, as also reported by Trouilhé et al. (2007), or human activities (local sources of organic pollution). In accordance with this general assumption, the maximum value of ammonium (reaching $2.1 \mathrm{mg} \cdot \mathrm{L}^{-1}$ of $\mathrm{NH}_{4}^{+}$) was observed in the Zákolanský brook near the city of Prague. The situation in this brook was more specific; there was also a low amount of dissolved oxygen $\left(2.8 \mathrm{mg} \cdot \mathrm{L}^{-1}\right)$ and such a situation is not suitable for aquatic organisms (Svobodová et al., 1987). However, higher pH (7.7) in this locality does not allow ammonium ion to change into free ammonium and that is probably why a numerous population of stone crayfish lives there. Although Svobodová et al. (2012) reported that ammonium ion is one of the most important parameters influencing distribution of indigenous crayfish species, Favaro 
et al. (2010) did not observe differences in the amount of ammonium in streams inhabited by stone crayfish and streams without its presence. We did not find a relation between either free ammonium or ammonium ion and the abundance of stone crayfish, even though the position of the Huníkovský brook in the RDA biplot indicates its divergence from other streams based on strong correlation of $\mathrm{NH}_{3}$ with the 2 nd axis of RDA. This position of the Huníkovský brook is hardly explainable, but it is supposed it is due to an inaccurate sample of water quality which affected the mean values of ammonium in this brook (Svobodová, oral communication).

The nitrates and nitrites are often products of nitrification of ammonium ion and are associated with organic pollution of sites (Pitter, 1999). In accordance with this fact, minimum values were found in natural localities without any influence of human activities (no nitrites in the Hrádecký and Bertínský brooks), while the highest average value of nitrites was found in the Radotínský brook $\left(0.46 \mathrm{mg} \cdot \mathrm{L}^{-1}\right)$ and the Zákolanský brook $\left(0.36 \mathrm{mg} \cdot \mathrm{L}^{-1}\right)$, the brooks under the influence of pollution from municipal waste. On average, $0.066 \mathrm{mg} \cdot \mathrm{L}^{-1}$ of nitrites were found in the localities inhabited by stone crayfish, which is consistent with data reported by Broquet et al. (2002) for $A$. pallipes. Nonetheless, this value is six times higher than that reported by Pârvulescu et al. (2011) in Romanian streams. A relationship between the abundance of stone crayfish and the amount of nitrite was not found, despite the above-mentioned extreme values.

The average value of nitrates reached $10.1 \mathrm{mg} \cdot \mathrm{L}^{-1}$, which is more than the value observed by Pârvulescu et al. (2011). It is also three times less than another value reported by Trouilhé et al. (2007). Nitrate values ranged between 0.24-45 mg.L-1 . Trouilhé et al. (2007) mentioned that Crayfish of the Austropotamobius genus is not sensitive to high daily $\mathrm{NO}_{3}^{-1}$ concentrations. A relationship between the abundance of stone crayfish and the amount of nitrate was found, and the parameter is included in the polynomial model which significantly influenced the abundance of crayfish (see Figure 5). However, the presented relationship is apparently illogical; it can be explained by correlation among the observed parameters (see Table I). We do not see nitrates as an important parameter in the observed range, in accordance with Jensen (1996) and Trouilhé et al. (2007), who reported nitrates to be a relatively non-toxic element.

The lowest individual values of calcium of around $4 \mathrm{mg} \cdot \mathrm{L}^{-1}$ were measured in Klabava, and the highest values in the Zákolanský Brook (163 mg. L-1 $)$, with extremes of over $170 \mathrm{mg} \cdot \mathrm{L}^{-1}$. This range extended the range observed by Kozák et al. (2002), but they only presented values from four sites with stone crayfish known at that time. In several streams numerous stone crayfish populations were found, even though calcium levels decreased regularly below the value reported as necessary for the development of crayfish exoskeleton calcification there (Bohl, 1987; Favaro et al., 2010; Foster, 1995; Rukke, 2002). The negative correlation between the amount of calcium and abundance of stone crayfish was proven using polynomial regression (see above). This seems to be inconsistent with the above-mentioned statements taking into account the need for calcification and seems to be illogical, and is probably due to a synergic effect. However, population density in streams with a high amount of calcium is affected in different ways, e.g. the Zbirožský brook is one of the biggest streams inhabited by stone crayfish with a high water level and sympatric occurrence of stone crayfish and noble crayfish - its competition and habitat features complicate catchability and therefore decrease observed abundance. The tributary of the Trojhorský brook was recently poisoned, and the Božkovský brook dries up periodically.

A positive correlation was calculated for sulphates, although this ion is considered not very important for crayfish Svobodová et al., 2008; Trouilhé et al., 2007). Favaro et al. (2010) reported the same fact and explained it as a result of the compounds $\mathrm{CaSO}_{4}$ and $\mathrm{MgSO}_{4}$, occurring commonly in streams.

The relationship between population density and the set of parameters (conductivity, nitrates, calcium and sulphates) was found. However, we suppose that the relations found should not be generalised as they are only the result of a relatively small set of localities inhabited by the stone crayfish, with highly correlated parameters, easy explainable by logically related facts (see discussion of particular parameters). 
In conclusion, this study indicates that $A$. torrentium is able to inhabit waters with a large range of physical and chemical parameters of the water without a fundamental influence on population densities, taking into account the fact that only a relatively small set of localities recently inhabited by the stone crayfish was investigated. The plasticity of crayfish of the genus Austropotamobius was also reported in the last decade (Demers, 2003; Favaro et al., 2010; Füreder and Reynolds, 2003; Svobodová et al., 2008; Trouilhé et al., 2007). Water parameters play an indisputable role as limiting ecological factors in uncommon concentration or factors increasing the probability of disease (Yildiz and Benli, 2004) and growth of individuals (Rukke, 2002). However, population densities are influenced much more by the types of habitats, habitat features (Trouilhé et al., 2007; Vlach et al., 2009a) or predation (Fischer et al., 2009).

\section{ACKNOWLEDGEMENTS}

This study was financially supported by the Ministry of the Environment of the Czech Republic within the framework of the project MZP 0002071101.

\section{REFERENCES}

Alexopoulos E., McCrohan C.R., Powell J.J., Jugdaohsingh R. and White K.N., 2003. Bioavailability and toxicity of freshly neutralised aluminium to the freshwater crayfish Pacifasticus leniusculus. Arch. Environ. Contam. Toxicol. , 45, 509-514.

Bohl E., 1987. Comparative studies on crayfish brooks in Bavaria (Astacus astacus L., Austropotamobius torrentium Schr.). Freshwater Crayfish, 7, 287-294.

Broquet T., Thibault M. and Neveu A., 2002. Distribution and habitat requirements of the white-clawed crayfish, Austropotamobius pallipes, in a stream from the Pays de Loire region, France: an experimental and descriptive study. Bull. Fr. Pêche Piscic., 367, 717-728.

Demers A., 2003. The water quality requirements of white-clawed crayfish, Austropotamobius pallipes (Lereboullet). Trinity College. University of Dublin, Dublin.

Demers A. and Reynolds J.D., 2002. A survey of the white-clawed crayfish, Austropotamobius pallipes, (Lereboullet), and of water quality in two catchments of eastern Ireland. Bull. Fr. Pêche Piscic., $367,729-740$.

Demers A., Souty-Grosset C., Trouilhé M.C., Füreder L., Renai B. and Gherardi F., 2006. Tolerance of three European native species of crayfish to hypoxia. Hydrobiologia, 260, 425-432.

Favaro L., Tirelli T. and Pessani D., 2010. The role of water chemistry in the distribution of Austropotamobius pallipes (Crustacea Decapoda Astacidae) in Piedmont (Italy). Comptes Rendus Biologies, 333, 68-75.

Fischer D., Pavluvčík P., Sedláček F. and Šálek M., 2009. Predation of the alien American mink, Mustela vison on native crayfish in middle-sized streams in central and western Bohemia. Folia Zool., 58, 45-56.

Foster J., 1995. Factors influencing the distribution and abundance of the crayfish Austropotamobius pallipes (Lereboullet) in Wales and the Marches, U.K. Freshwater Crayfish, 8, 78-98.

Füreder L. and Reynolds J.D., 2003. Is Austropotamobius pallipes a good bioindicator? . Bull. Fr. Pêche Piscic., 157-163.

Füreder L., Oberkofler B., Hanel R., Leiter J. and Thaler B., 2003. The freshwater crayfish Austropotamobius pallipes in South Tyrol: heritage species and bioindicator. Bull. Fr. Pêche Piscic., 370-371, 81-95.

Füreder L., Gherardi F. and Souty-Grosset C., 2010. Austropotamobius torrentium. In: IUCN 2010, www.iucnredlist.org. Downloaded 26 November 2010.

Grandjean F., Bramard M. and Souty-Grosset C., 1996. Distribution and proposals for the conservation of Austropotamobius pallipes pallipes in a French department. Freshwater Crayfish, 11, 655-664.

Guner U., 2007. Freshwater crayfish Astacus leptodactylus (Eschscholtz, 1823) accumulates and depurates copper. Environ. Monit. Assess., 133, 365-369.

Holdich D.M., 2002. Distribution of crayfish in Europe and some adjoining countries. Bull. Fr. Pêche Piscic., 367, 611-650. 
Jeberg M.V. and Jensen F.B., 1994. Extracellular and intracellular ionic changes in crayfish Astacus astacus exposed to nitrite at two acclimation temperatures. Aquat. Toxicol., 29, 65-72.

Jensen F.B., 1996. Uptake, elimination and effects of nitrite and nitrate in freshwater crayfish (Astacus astacus). Aquat. Toxicol. , 34, 95-104.

Kappus B., Peissner T. and Raver-Jost C., 1999. Distribution and habitat conditions of crayfish populations in the urban freshwater systems of Stuttgart (Baden-Württenberg, Germany). Freshwater Crayfish, 12, 778-785.

Kozák P., Policar T., Ďuriš Z. and Theimer J., 2001. Rak kamenac Austropotamobius torrentium (Schrank) v Ceske republice (The stone crayfish Austropotamobius torrentium (Schrank) in the Czech Republic). Casopis SZM, 50, 85-93.

Kozák P., Ďuriš Z. and Policar T., 2002. The stone crayfish Austropotamobius torrentium (Schrank) in the Czech republic. Knowl. Managt. Aquatic Ecosyst., 367, 707-713.

Kozák P., Máchová J. and Policar T., 2005. The effect of chloride content in water on the toxicity of sodium nitrite for spiny-cheek crayfish (Orconectes limosus Raf.). Bull. Fr. Pêche Piscic., 376-377, 705-713.

Lachat G. and Laurent P.J., 1987. The habitats of Astacus astacus L. and Austropotamobius pallipes Lere. in the Morvan. Freshwater Crayfish, 7, 61-68.

Machino Y. and Füreder L., 2005. How to find a stone crayfish Austropotamobius torrentium (Schrank, 1803): A biogeographic study in Europe. Bull. Fr. Pêche Piscic., 376-377, 507-517.

Pârvulescu L., Pacioglu O. and Hamchevici C., 2011. The assessment of the habitat and water quality requirements of the stone crayfish (Austropotamobius torrentium) and noble crayfish (Astacus astacus) species in the rivers from the Anina Mountains (SW Romania). Knowl. Managt. Aquatic Ecosyst., 401, 03.

Pitter P., 1999. Hydrochemistry. VŠCHT, Prag. 568 p.

Renz M. and Breithaupt T., 2000. Habitat use of the crayfish Austropotamobius torrentium in small brooks and in Lake Constance, Southern Germany. Bull. Fr. Pêche Piscic., 356, 139-154.

Rukke N.A., 2002. Effects of low calcium concentrations on two common freshwater crustaceans, Gammarus lacustris and Astacus astacus. Funct. Ecol., 16, 357-366.

Seiler S.M. and Turner A.M., 2004. Growth and population size of crayfish in headwater streams: individual- and higher-level consequences of acidification. Freshw. Biol., 49, 870-881.

Sint D. and Füreder L., 2004. Reintroduction of Astacus astacus L. in East Tyrol, Austria. Bull. Fr. Pêche Piscic., 372-373, 301-314.

Souty-Grosset C., Holdich D.M., Noël P.Y., Reynolds J.D. and Haffner P. (eds.), 2006. Atlas of Crayfish in Europe. Patrimoines Naturels 64. Muséum National d'Histoire Naturelle, Paris, 187 p.

Štambergová M., Svobodová J. and Kozubíková E., 2009. Raci v České republice. AOPK ČR, Praha.

Svobodová J., Štambergová M., Vlach P., Picek J., Douda K. and Beránková, M., 2008. The impact of the water quality on the crayfish population in the Czech Republic, comparison with legislation of the Czech Republic. VTEI, 50, 1-5 (in czech with english summary).

Svobodová J., Douda K., Štambergová M., Picek J., Vlach P. and Fischer D., 2012. The relationship between water quality and indigenous and alien crayfish distribution in the Czech Republic: patterns and conservation implications. Aquatic Conserv. Mar. Freshw. Ecosyst., 22, 776-786 .

Svobodová Z., Gelnarová J., Justýn J., Krupauer V., Simanov L., Valentová V., Vykusová B. and Wohlgemuth E., 1987. Toxicology of water organisms. SZN, Prag. 231 p. (in czech).

Trouilhé M.C., Souty-Grosset C., Grandjean F. and Parinet B., 2007. Physical and chemical water requirements of the white-clawed crayfish (Austropotamobius pallipes) in western France. Aquatic Conserv. Mar. Freshw. Ecosyst., 17, 520-538.

Vlach P., Fischer D. and Hulec L., 2009a. Microhabitat preference of the stone crayfish Austropotamobius torrentium (Schrank, 1803). Knowl. Managt. Aquatic Ecosyst., 384-395, 15.

Vlach P., Hulec L. and Fischer D., 2009b. Recent distribution, population densities and ecological requirements of the stone crayfish (Austropotamobius torrentium) in the Czech Republic. Knowl. Managt. Aquatic Ecosyst., 384-395, 13.

Ward R.J., McCrohan C.R. and White K.N., 2006. Influence of aqueous aluminium on the immune system of the freshwater crayfish Pacifasticus leniusculus. Aquat. Toxicol., 77, 222-228.

Wright J.F., Sutcliffe D.V. and Furse M.F. (eds.), 2000. Assessing the biological quality of fresh waters, RIVPACS, and other techniques. Freshwater Biological Association, East Stoke, $373 \mathrm{p}$.

Yildiz H.Y. and Benli A.C.K., 2004. Nitrite toxicity to crayfish, Astacus leptodactylus, the effects of sublethal nitrite exposure on hemolymph nitrite, total hemocyte counts, and hemolymph glucose. Ecotoxicol. Environ. Saf., 59, 370-375. 